\title{
A 3D view of genome rearrangements
}

Somatic structural genome alterations such as changes in copy number or chromosomal rearrangements can promote genome instability and cancer development, and identification of these mutations is crucial to diagnose cancer early and provide targeted therapies. Now, Harewood et al. report the use of $\mathrm{Hi}-\mathrm{C}$ ( $\mathrm{a}$ high-throughput derivative of the chromosome conformation capture (3C) technology) for the detection of both balanced and unbalanced chromosomal rearrangements in primary human tumour samples. The same data can also be used to determine copy number profiles.

The detection of somatic structural genome alterations in cancer has enormously increased in efficiency and resolution with the development of next-generation sequencing technologies. However, mapping rearrangements in repetitive regions or detecting balanced rearrangements (where the genomic rearrangement does not lead to an overall loss or gain of genetic material), such as reciprocal translocations and inversions, has remained challenging.

The authors generated in-nucleus Hi-C heat maps for two human lymphoblastoid cell lines with known chromosomal translocations between chromosomes 11 and 22. Distinct blocks visible on the heat map reflect unusually strong 'long-range' cis or trans interactions, which arise when chromosomal rearrangements bring together distal regions of the same or different chromosomes, respectively. The first heat map for a cell line containing an unbalanced rearrangement (where there is either loss or gain of genetic material) showed one distinct block that mapped to known breakpoints. By contrast, a second cell line containing a balanced rearrangement showed two blocks that were joined at the point of strongest contacts, corresponding to known chromosomal breakpoints, which is indicative of a reciprocal translocation.
It was previously reported that Hi-C can detect novel chromosomal rearrangements in cell lines. Confirming these findings, the authors performed in-nucleus $\mathrm{Hi}-\mathrm{C}$ on a transformed mouse cell line and identified three unbalanced translocations from clear single blocks of strong contacts between sequences on chromosomes 3 and 10, 10 and 16 , and $\mathrm{X}$ and 8 . These translocations could subsequently be validated using dual-colour DNA fluorescence in situ hybridization (FISH).

Demonstrating the potential of $\mathrm{Hi}-\mathrm{C}$ for the identification of unknown chromosomal rearrangements in the clinic, the authors applied the Hi-C approach to six human brain tumours, providing an in-depth characterization of the different rearrangements in each tumour. Even complex chromosomal rearrangements involving more than two chromosomes could be defined, and breakpoints were mappable to base pair resolution.

Using the Hi-C data obtained from the six brain tumours, the authors were able to determine copy number alterations using the QDNAseq pipeline, which extracts copy number information from DNA samples without the need for a reference.

Combining the ability to determine structural aberrations with an overview on how whole chromosomes are involved in rearrangements, rather than focusing only on breakpoint regions, will be useful for the identification and understanding of complex chromosomal rearrangements in cancer. By precluding the need for deep sequencing, the insights gained by $\mathrm{Hi}-\mathrm{C}$ will also come at a considerably lower cost than with current approaches.

\section{Linda Koch}

ORIGINAL ARTICLE Harewood, L. et al. Hi-C as a tool for precise detection and characterisation of chromosomal rearrangements and copy number variation in human tumours. Genome Biol. http:// dx.doi.org/10.1186/s13059-017-1253-8 (2017) breakpoints

were

mappable

to base pair

resolution 